\title{
Holographic complexity of cold hyperbolic black holes
}

José L.F. Barbón and Javier Martín-García

Instituto de Física Teórica IFT UAM/CSIC,

Calle Nicolas Cabrera 13, UAM, Cantoblanco 28049. Madrid, Spain

E-mail: jose.barbon@uam.es, javoxmg@gmail.com

ABSTRACT: AdS black holes with hyperbolic horizons provide strong-coupling descriptions of thermal CFT states on hyperboloids. The low-temperature limit of these systems is peculiar. In this note we show that, in addition to a large ground state degeneracy, these states also have an anomalously large holographic complexity, scaling logarithmically with the temperature. We speculate on whether this fact generalizes to other systems whose extreme infrared regime is formally controlled by Conformal Quantum Mechanics, such as various instances of near-extremal charged black holes.

KEYwords: Duality in Gauge Field Theories, AdS-CFT Correspondence, Black Holes

ArXiv ePrint: 1510.00349 


\section{Contents}

1 Introduction 1

2 Cold hyperbolic horizons $\quad 3$

3 Estimating holographic complexity $\quad 4$

3.1 High temperatures $\quad 6$

$\begin{array}{lll}3.2 & \text { Low temperatures } & 6\end{array}$

4 Conclusions $\quad 8$

\section{Introduction}

Computational complexity has been recently proposed as a new entry in the holographic dictionary between space-time geometry and quantum entanglements. Besides the total amount of entanglement, measured by the entanglement entropy and loosely associated to the 'connectivity' of emergent space-time [1-4], the sheer amount of emergent space seems to be associated to the degree of complexity of the entanglement structure, measured with respect to a set of elementary entanglement operations.

One motivation for this correspondence arises form the tensor network models of geometry [5-11]. In this picture, a notion of computational complexity of the state can be associated to the volume of the tensor network making it plausible that a quantitative volume/complexity relation may exist (cf. [12-16]):

$$
C(t) \propto \frac{\operatorname{Vol}\left(\Sigma_{t}\right)}{G},
$$

where $\Sigma_{t}$ is a codimension-one space-like section of the bulk with extremal volume, parametrized by some proper-time coordinate $t .^{1}$

A qualitative test of (1.1) is offered by the analysis of eternal AdS black holes, interpreted as thermofield-double states entangling two decoupled CFTs defined on spheres $[1,4,11]$. In this case, $t$ can be chosen as the standard AdS time in the global static frame, and the non-trivial time dependence of (1.1) comes from the portion of $\Sigma_{t}$ lying in the interior of the black hole. There is a saturating surface which represents a linearly growing wormhole section, so that one finds

$$
\frac{d C}{d t} \sim T S
$$

for $t \gg T^{-1}$, where $T$ is the temperature and $S$ the entropy of the eternal black hole. If the wormhole is approximated by a flat tensor network, this formula can be interpreted

\footnotetext{
${ }^{1}$ See [17] and [18] for further discussion of ab initio approaches to holographic complexity.
} 
as the growth of an effective quantum circuit of $S$ Qbits [11, 12], a representation which makes contact with the traditional definition of computational complexity in quantum information theory. ${ }^{2}$

The calculation of (1.2) within the eternal black hole geometry assumes implicitly that $T$ is sufficiently large to neglect finite-size effects. For standard AdS black holes, this means that $T \ell \gg 1$ where $\ell$ has the dual interpretation as the $\mathrm{AdS}_{d+1}$ radius of curvature in the bulk and also the radius of the $(d-1)$-sphere where the $d$-dimensional CFTs are defined. In this particular case the $T \ell \ll 1$ limit is on the other side of the Hawking-Page transition, and the $O(1 / G)$ contribution to the complexity must be calculated in the vacuum AdS manifold, giving no contribution at this order to (1.2).

Alternatively, we can remove finite-size effects by working with black branes of noncompact horizon, where all integrated quantities, such as entropy and complexity, are extensive in the CFT volume. In this case we implicitly refer to a 'complexity density'. ${ }^{3}$ Black-brane metrics have the general form

$$
d s^{2}=-f(r) d t^{2}+\frac{d r^{2}}{f(r)}+\frac{r^{2}}{\ell^{2}} d L^{2}
$$

where $d L^{2}$ stands for the spatial CFT metric and $f(r)$ has a non-degenerate horizon at $r=r_{0}$ with Hawking temperature $T$, i.e. $f(r) \approx 4 \pi T\left(r-r_{0}\right)$ near the horizon. We also require vacuum asymptotics $f(r) \sim r^{2} / \ell^{2}$ as $r \rightarrow \infty$.

There are standard solutions given by

$$
f(r)=k+\frac{r^{2}}{\ell^{2}}-\frac{\mu}{r^{d-2}} .
$$

for flat $(k=0)$ and hyperbolic $(k=-1)$ CFT metrics. In the first case the usual UV/IR relation $r_{0} \sim \ell^{2} T$ holds down to zero temperature, with the entropy vanishing as $T^{d-1}$. In the second case, the CFT lives on a hyperboloid of curvature radius $\ell$. This system has exotic properties at low temperatures [23], in particular a gross violation of the third law of thermodynamics

$$
\lim _{T \rightarrow 0} S \longrightarrow S_{0}=N_{*} V \ell^{1-d}
$$

where $N_{*} \sim \ell^{d-1} / G \gg 1$ is the effective number of 'species' in the strongly-coupled CFT.

The purpose of this note is to study some properties of the holographic complexity, as defined by the ansatz (1.1), in such degenerate systems. In particular, we shall consider the concrete case of thermofield double states for pairs of CFTs on hyperboloids, as defined by AdS hyperbolic black holes [24-33]. We begin in section 2 with a review of the relevant geometries and we continue in section 3 with the approximate calculation of the complexity.

\footnotetext{
${ }^{2}$ The conditions for the tensor network to really represent a smooth wormhole geometry are not well understood [19-21].

${ }^{3}$ This is in principle different from the strict definition of a notion of complexity for a subsystem, see [22] for discussions in this direction.
} 


\section{Cold hyperbolic horizons}

We consider the black-brane metric (1.3) with hyperbolic horizon geometry, which has the interpretation of a thermal state for a CFT on a spatial $(d-1)$-dimensional hyperboloid $\mathrm{H}_{d-1}$ :

$$
f(r)=-1+r^{2}-\frac{m}{r^{d-2}}, \quad d L^{2}=d \mathrm{H}_{d-1}^{2}=d \chi^{2}+\sinh ^{2}(\chi) d \Omega_{d-2},
$$

where we measure lengths in units of the curvature radius $\ell=1$. Alternatively, the maximally extended geometry can be interpreted, following [1], as dual to a thermofield double state on the direct product of two copies of the CFT on respective hyperboloids.

The mass parameter $m$ is related to the horizon radius $r_{0}$ by

$$
m=r_{0}^{d-2}\left(r_{0}^{2}-1\right)
$$

and the Hawking temperature is given by

$$
T=\frac{r_{0}}{4 \pi}\left(d-\frac{d-2}{r_{0}^{2}}\right) .
$$

The case of vanishing mass parameter is special, corresponding to $T=1 / 2 \pi$, the Rindler temperature. At this particular value the metric is nothing but a Rindler patch of the vacuum AdS manifold. The corresponding thermofield double on two decoupled hyperboloids is conformally equivalent to the hemispherical decomposition of a single copy of the CFT on a unit sphere (cf. for example [2,3]). The same bulk geometries can also be interpreted as computing properties of a certain entangled state on two static patches of a de Sitter CFT (cf. for example [34]).

Here, we are more interested in the $T \rightarrow 0$ limit, where the horizon drops to a minimum radius

$$
r_{c}=\sqrt{\frac{d-2}{d}}
$$

corresponding to a negative mass parameter

$$
m_{c}=-\frac{2}{d}\left(\frac{d-2}{d}\right)^{\frac{d-2}{2}} .
$$

In this extremal case the function $f(r)$ develops a double zero at the horizon, namely in the vicinity of $r=r_{c}$ we can write

$$
f(r)_{T=0}=d \cdot\left(r-r_{c}\right)^{2}+\ldots
$$

where the dots stand for terms of order $\left(r-r_{c}\right)^{3}$ or higher. This suggests that we can parametrize the low-temperature geometries in terms of the radial variable $\rho=r-r_{c}$. Then, to first non-trivial order in $\rho$ and $\rho_{0}=r_{0}-r_{c}$ we have

$$
f(r) \approx d \cdot\left(\rho^{2}-\rho_{0}^{2}\right)+\ldots,
$$


an approximation good for $\rho_{0} \leq \rho \ll r_{c}$. The low-temperature horizon sits at $\rho=\rho_{0} \approx$ $2 \pi T / d+O\left(T^{2}\right)$.

The black AdS geometry changes character in the vicinity of the minimum radius $r \sim r_{c}$, so that the region $\rho_{0} \ll \rho \ll r_{c}$, arising at very low temperatures, is approximately described by $\mathrm{AdS}_{1+1} \times \mathrm{H}_{d-1}$, i.e. the hyperbolic 'space' decouples from an asymptotic $\mathrm{AdS}_{2}$ factor. The corresponding curvature radii are given by

$$
\ell_{\mathrm{AdS}_{2}}=\frac{1}{\sqrt{d}}, \quad \ell_{\mathrm{H}_{\mathrm{IR}}}=\sqrt{\frac{d-2}{d}},
$$

measured in units $\ell=1$. We will refer to this factorized geometry as the CQM region, to signify the formal $\mathrm{AdS}_{2} / \mathrm{CFT}_{1}$ duality to some hypothetical Conformal Quantum Mechanical (CQM) system that would describe the deep infrared regime.

\section{Estimating holographic complexity}

Following the formula (1.1), we compute the holographic complexity as the volume of extremal codimension-one surfaces in the given geometry, parametrized by the static asymptotic time variable. We shall also parametrize the absolute normalization of (1.1) as differing from the RT formula of entanglement entropy $[35,36]$ by a factor $\alpha$.

The exact variational problem is complicated, but a useful order-of-magnitude estimate can be obtained by an approximate description of the full metric (1.3), according to a piecewise approximation for the function $f(r)$. For $r \gg r_{0}$ we can approximate the metric by the vacuum $\mathrm{AdS}_{d+1}$ solution. In the near-horizon region $r_{0}<r<r_{R}$, with $r_{R}$ an $O(1)$ multiple of $r_{0}$, we can take the Rindler approximation, whereby the metric is expressed as a product of two-dimensional flat space and the horizon:

$$
d s_{\text {Rindler }}^{2} \approx-\left(d X^{0}\right)^{2}+\left(d X^{1}\right)^{2}+r_{0}^{2} d \mathrm{H}_{d-1}^{2},
$$

where

$$
X^{0}=\sqrt{\frac{r-r_{0}}{\pi T}} \sinh (2 \pi T t), \quad X^{1}=\sqrt{\frac{r-r_{0}}{\pi T}} \cosh (2 \pi T t),
$$

a change of variables valid for $r>r_{0}$ on one of the asymptotic regions. Finally, the interior geometry is parametrized in Schwarzschild coordinates $(r, t)$, formally continued to $r<r_{0}$, with $r$ now denoting a time-like coordinate and $t$ a space-like one. There is an analogous extension of the Rindler patch to the interior, with the analogous change of variables

$$
X^{0}=\sqrt{\frac{r_{0}-r}{\pi T}} \cosh (2 \pi T t), \quad X^{1}=\sqrt{\frac{r_{0}-r}{\pi T}} \sinh (2 \pi T t) .
$$

Within this prescription we view the portion of the extremal surface lying outside the horizon as composed of two pieces: an asymptotic component $\Sigma_{\mathrm{UV}}$ which is well approximated by a constant $t$ surface in $\mathrm{AdS}_{d+2}$, and a 'Rindler piece' $\Sigma_{\mathrm{R}}$, parametrized by a curve on the $\left(X^{0}, X^{1}\right)$ plane of (3.1). Within the Rindler patch, local volume for fixed $X^{1}$ interval is maximized by the $X^{0}=$ constant surfaces, and thus we take this ansatz for $\Sigma_{\mathrm{R}}$. For $t=0$, this is all there is, since the extremal surface is just the $t=0$ section of the 


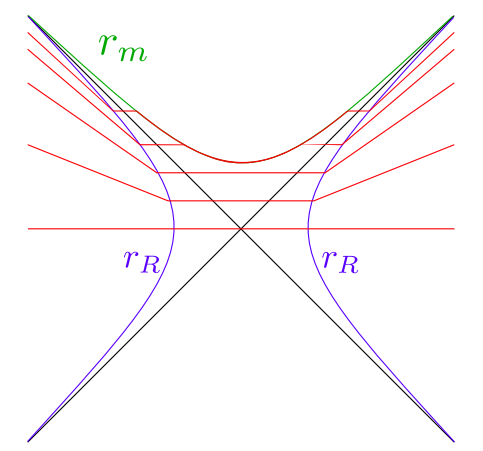

Figure 1. Piecewise decomposition of $\Sigma_{t}$, represented by space like sections at different times. For generic times, straight tilted segments correspond to $\Sigma_{\mathrm{UV}}$, horizontal segments give $\Sigma_{\mathrm{R}}$ and hyperbolic segments on the $r=r_{m}$ surface correspond to $\Sigma_{\mathrm{WH}}$. The $t=0$ surface lacks an interior component. As $t$ increases from zero, an interior component begins to develop gradually, as $\Sigma_{\mathrm{R}}$ eventually transmutes into $\Sigma_{R} \cup \Sigma_{\mathrm{WH}}$.

extended geometry, with the two exterior geometries glued by the horizon. However, as $t$ grows, the surface enters the horizon at higher values of $X^{0}$ and tends to extend through the interior patch of the black brane geometry. The $X^{0}=$ constant ansatz continues to be reasonable as long as the complete surface stays inside the interior Rindler region. Since the only length scale controlling the width of the Rindler region is $T^{-1}$, the approximation $X^{0}=$ constant must break down for large times, $t \gg T^{-1}$.

At very long times, there is a natural answer for the variational problem in the interior, since the surfaces $r=$ constant are invariant under the $t$-translation isometry. The volume of a $\Delta t$ portion of such $r=$ constant surfaces is proportional to

$$
\Delta t r^{d-1} \sqrt{|f(r)|}
$$

so that stationary points $r_{m}$ of this function determine extremal surfaces far from the 'exit point', i.e. for large $\Delta t$. In all cases studied in this paper, one finds $\left|r_{0}-r_{m}\right| \sim\left|r_{R}-r_{0}\right|$, implying that $r_{m}$ is always close to the inner edge of the Rindler region and, in particular, it is roughly symmetrical of the $r=r_{R}$ surface by a reflection through the horizon (see figure 1). As a consequence, the 'exit point' from the $r=r_{m}$ surface is approximately given by $t_{\text {exit }} \approx t$, where $t$ is the time label of the exterior asymptotic surface $\Sigma_{\mathrm{UV}}$.

The approximate ansatz for the extremal surface is thus $\Sigma_{\mathrm{WH}} \cup \Sigma_{\mathrm{R}} \cup \Sigma_{\mathrm{UV}}$, where $\Sigma_{\mathrm{WH}}$ is the $r=r_{m}$ surface along the 'wormhole' in the interior, cut off at $t_{\text {exit }} \sim t$, with total $t$-length of order $\Delta t \sim 2 t$.

Within this construction, the volume of $\Sigma_{\mathrm{UV}}$ is independent of $t$, whereas the volume of $\Sigma_{\mathrm{R}}$ vanishes at large $t$, being delimited by two curves (interior and exterior) asymptotic to the same horizon. Therefore, the rate of growth of the complexity is controlled by $\Sigma_{\mathrm{WH}}$ at large times. A graphical representation of the piecewise decomposition of $\Sigma_{t}$ is shown in figure 1. 


\subsection{High temperatures}

At high temperatures $T \gg 1$ we have $f(r) \approx r^{2}-r_{0}^{d} / r^{d-2}$ and $r_{0} \approx 4 \pi T / d$. Evaluating the volume of $\Sigma_{t}$, we find the standard result (1.2) for the long-time growth rate, with $S \sim N_{*} V T^{d-1}$ the high temperature entropy of the large- $N_{*}$ CFT on the hyperboloid. At $t=0$, we can distinguish two qualitatively different contributions. First we have the UV contribution of $\Sigma_{\mathrm{UV}}$,

$$
C_{\mathrm{UV}}=2 \alpha \frac{V}{4 G} \int_{r_{R}}^{r_{\Lambda}} \frac{d r r^{d-1}}{\sqrt{f(r)}} \sim N_{*} V\left(\Lambda^{d-1}-T^{d-1}\right),
$$

where we can as well neglect the $T$-dependent term coming from the lower limit of the integral, since we are assuming $\Lambda \gg T$. Second, we have a threshold contribution coming form $\Sigma_{\mathrm{R}}$ :

$$
\left.C_{\mathrm{R}}\right|_{t=0}=2 \alpha \frac{V}{4 G} \int_{r_{0}}^{r_{R}} \frac{d r r^{d-1}}{\sqrt{f(r)}} \sim N_{*} V T^{d-1} \sim S,
$$

where we have used the Rindler approximation to the metric to estimate the integral in order of magnitude. In this expression, as well as others that follow, the matching ambiguity coming from the precise location of $r_{R}$ and the various errors from the piecewise matchings of $\Sigma_{t}$ can be estimated by shifting $r_{R}$ an amount of $O(1)$, resulting in an additive ambiguity of order $S$ for $C_{\mathrm{R}}$.

The UV contribution to the complexity is constant in time. Denoting the rest of the complexity by $\Delta C(t)=C(t)-C_{\mathrm{UV}}$ we find the following behavior at high temperatures:

$$
\Delta C(t)=O(S) \text { for } t<T^{-1}, \quad \Delta C(t) \sim S T t \text { for } t \gg T^{-1} .
$$

\subsection{Low temperatures}

Our main interest is the low-temperature regime, $T \ll 1$ in $\ell=1$ units, where the thermodynamics becomes more exotic. In evaluating the volume of extremal surfaces, we must distinguish the qualitatively different regions of the bulk geometry, namely for $r \gg r_{c}$ we have an approximately $\mathrm{AdS}_{d+1}$ geometry with a time slicing adapted to the $\mathbf{R} \times \mathrm{H}_{d-1}$ CFT frame, and for $\rho_{0} \ll \rho \ll r_{c}$ we have a $\mathrm{AdS}_{1+1} \times \mathrm{H}_{d-1}$ geometry. Accordingly, the codimension-one surfaces split as (see figure 2)

$$
\Sigma_{t} \sim \Sigma_{\mathrm{WH}} \cup \Sigma_{\mathrm{R}} \cup \Sigma_{\mathrm{CQM}} \cup \Sigma_{\mathrm{UV}}
$$

Here $\Sigma_{\mathrm{UV}}$ extends for $r \gg r_{c}$. The new portion extending along the $\mathrm{AdS}_{1+1}$ radial slice $\rho_{0} \ll \rho \ll r_{c}$ will be denoted $\Sigma_{\mathrm{CQM}}$. Finally, in the deep infrared region we have the Rindler portion $\Sigma_{\mathrm{R}}$ given by the interval $\rho_{0}<\rho<\rho_{R}$, with $\rho_{R}$ an $O(1)$ multiple of $\rho_{0}$. In the interior we find the wormhole portion $\Sigma_{\mathrm{WH}}$ along $r=r_{m}$. For all partitions except $\Sigma_{\mathrm{UV}}$ we can regard the hyperbolic $\mathrm{H}_{d-1}$ factor as an spectator.

We first discuss the situation at $t=0$, where $\Sigma_{\mathrm{WH}}$ is absent. The contribution from $\Sigma_{\mathrm{UV}}$ is the standard $N_{*} V \Lambda^{d-1}$. The contribution from $\Sigma_{\mathrm{CQM}}$ is interesting because the complexity picks equal contributions for every region of the CQM region,

$$
C_{\mathrm{CQM}}(0) \approx 2 \alpha \frac{r_{c}^{d-1} V}{4 G} \int_{\rho_{R}}^{r_{c}} \frac{1}{\sqrt{d}} \frac{d \rho}{\rho}=\frac{2 \alpha}{\sqrt{d}} S_{0} \log (1 / T),
$$




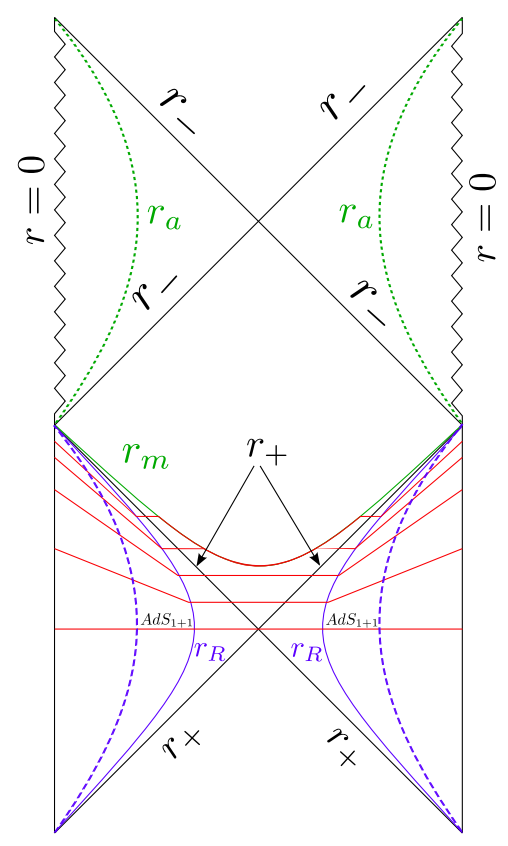

Figure 2. The global structure of the hyperbolic black hole interior is similar to that of charged black holes. There is an inner horizon $r_{-}$which separates the space-like and time-like character of fixed- $r$ surfaces in the interior. For instance, one has the inequalities $0<r_{a}<r_{-}<r_{m}<r_{+}=$ $r_{0}<r_{R}$. The intermediate $\mathrm{AdS}_{2}$ region develops only for low temperatures.

leading to a logarithm with a characteristic coefficient controlled by the zero-temperature entropy of the system. The $O(1)$ ambiguities at the endpoints of the integral amount to an additive error of order $S_{0}$. Notice however that the coefficient of the logarithm, given by $2 \alpha S_{0} / \sqrt{d}$, is robust in the low $T$ limit.

Finally, the Rindler contribution coming from $\Sigma_{R}$ is of order

$$
C_{\mathrm{R}}(0) \approx 2 \alpha \frac{r_{c}^{d-1} V}{4 G} \int_{\rho_{0}}^{\rho_{R}} \frac{1}{\sqrt{d}} \frac{d \rho}{\sqrt{\rho^{2}-\rho_{0}^{2}}} \sim S_{0}
$$

where the matching errors are also of order $S_{0}$.

As before, the exterior surfaces in both the CQM and UV regions have a timeindependent volume. Hence the time development of the complexity proceeds by the gradual deformation of $\Sigma_{\mathrm{R}}$ into $\Sigma_{\mathrm{WH}} \cup \Sigma_{\mathrm{R}}$. As can be seen from figure 2 , the volume of $\Sigma_{\mathrm{R}}$ is negligible at large times, whereas that of $\Sigma_{\mathrm{WH}}$ is controlled by the local maximum of $r^{d-1} \sqrt{|f(r)|}$. Since we are working at very low temperatures, it is tempting to pick the $O(1)$ radius $r=r_{a}$ which maximizes the $T=0$ function

$$
r^{d-1} \sqrt{\left|1-r^{2}+\frac{m_{c}}{r^{d-2}}\right|} .
$$

However, there is a subtlety. This $O(1)$ maximum at $r=r_{a}$ survives for small but non-zero $T$, but in fact we have $f\left(r_{a}\right)>0$, implying that $r=r_{a}$ is a time-like surface (shown in figure 2). It turns out that there is a small $T$-dependent local maximum of $r^{d-1} \sqrt{|f(r)|}$, 


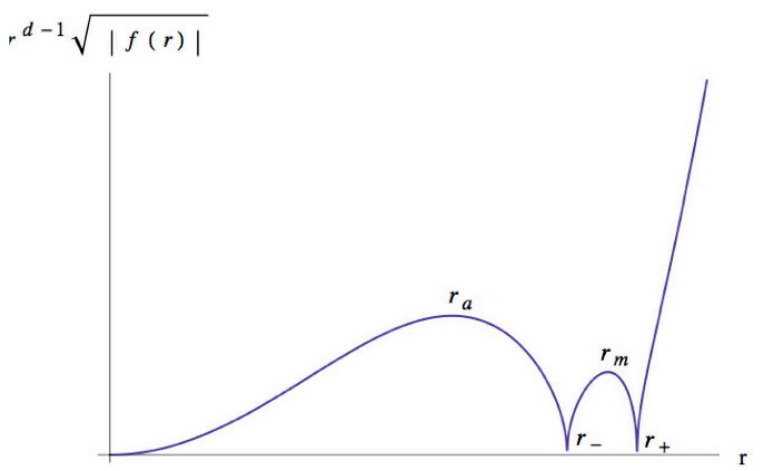

Figure 3. The function $r^{d-1} \sqrt{|f(r)|}$ at low temperatures, showing the small maximum of $O(T)$ at $r=r_{m}$ in the near-horizon (Rindler) region, and the $O(1)$ maximum at $r=r_{a}$. In the high- $T$ regime the Rindler bump grows larger than the local maximum at $r=r_{a}$, which becomes a small detail near the singularity.

with height of $O(T)$, within the interior Rindler region (see figure 3). The corresponding $r=r_{m}$ surface is space-like, since $f\left(r_{m}\right)<0$. In this regime the function to be maximized is approximately given by

$$
\left(r_{c}+\rho\right)^{d-1} \sqrt{d\left|\rho_{0}^{2}-\rho^{2}\right|},
$$

which is maximized close to $\rho_{m}=0$, so that the WH surface is given by $\rho \approx 0$. Again, it is roughly the symmetrical of the $\rho=\rho_{R}$ surface by a reflection with respect to the horizon, implying that $t_{\text {exit }} \sim t$ and thus a 'wormhole length' of order $\Delta t \approx 2 t$. The resulting large $t$ complexity is

$$
C_{\mathrm{WH}}(t)=2 t \alpha \frac{V r_{c}^{d-1}}{4 G} \sqrt{d} \rho_{0} \approx \alpha \frac{4 \pi T}{\sqrt{d}} S_{0} t
$$

Grouping together these results and restoring the curvature radius $\ell$, we find a total lowtemperature subtracted complexity given by

$$
\Delta C(t) \approx \frac{2 \alpha}{\sqrt{d}} S_{0} \log (1 / \ell T), \text { for } t<T^{-1},
$$

at small times and

$$
\Delta C(t) \approx \frac{2 \alpha}{\sqrt{d}} S_{0} \log (1 / \ell T)+\frac{4 \pi \alpha}{\sqrt{d}} S_{0} T t \text { for } t \gg T^{-1} .
$$

at long times. It should be noted that, while we have kept the coefficient found in (3.9), it must be understood as an estimate with $O\left(S_{0}\right)$ additive ambiguities, unlike the coefficient of the time-independent logarithmic term, which is a robust prediction for the strongly coupled CFT.

\section{Conclusions}

We have studied the structure of low-temperature thermofield double states in strongly coupled CFTs defined on hyperboloids. In particular, we have focused on properties characterized by the gravitational description in terms of AdS hyperbolic black holes. We have 
found that, in addition to the known zero-temperature entropy of order $N_{*}$, these states have a large holographic complexity, as measured by extremal bulk volumes.

The low-temperature geometry develops a $\mathrm{AdS}_{2}$ throat contributing a timeindependent complexity of order

$$
C_{\mathrm{CQM}}=\frac{2 \alpha S_{0}}{\sqrt{d}} \log (1 / T \ell)+O\left(S_{0}\right)
$$

where $\ell$ is the radius of curvature of the hyperboloid and $S_{0}$ is the zero-temperature limit of the entropy. This expression was derived up to additive ambiguities of $O\left(S_{0}\right)$, but the coefficient of the logarithm is a reliable strong-coupling prediction in the low- $T$ limit, once we define the absolute normalization of the complexity, which entails fixing the value of $\alpha$.

This means that tensor network representations of these states should incorporate this logarithmic tube. It would be interesting to find an explicit tensor model realizing this fact. Perhaps these ideas can be checked at weak coupling, searching for vestiges of the large low-temperature complexity directly in the perturbative field theory wave functions.

Since the behavior (4.1) is controlled by the emergence of the $\mathrm{AdS}_{2}$ throat, it is tempting to take it at face value, as a general property of any near-extremal geometry of ReissnerNordstrom type. This includes the benchmark model of AdS/CMT, the near extremal charged $\mathrm{AdS}_{4}$ black brane, with either chemical potential $\mu$ or magnetic field $B$ (cf. [37] for a review). In those systems, the same expression (4.1) follows, with the substitution of the curvature scale $1 / \ell$ by an effective mass of the order of $\mu$ and/or $\sqrt{B}$ (cf. [38] for a discussion of peculiar properties of entanglement entropy in these systems). On the other hand, it is also known that consistent string theory embeddings of these finite-density systems tend to show perturbative instabilities, the near extremal black holes being unstable to the condensation of clouds of classical charged hair (see [18] for a discussion of possible relations to complexity).

The low-temperature hyperbolic black holes studied in this note show no sign of any perturbative instability when embedded in string theory, but they are not free from potential non-perturbative instabilities. As shown in [39], if these black holes carry $N$ units of RR charge, they can super-radiate it spontaneously, causing the fragmentation of the black hole by brane emission (see also [40-43]). This process was studied in [39] for compact hyperbolic horizons, but it may take place in the present non-compact set up by nucleation of critical bubbles just hovering above the horizon, leading to a sort of condensation of RR hair. It would be interesting to further clarify these issues and their possible impact on the prediction (4.1).

\section{Acknowledgments}

We thank E. Rabinovici for discussions. This work was partially supported by MINECO and FEDER under a grant FPA2012-32828, and the spanish MINECO Centro de Excelencia Severo Ochoa Program under grant SEV-2012-0249. 
Open Access. This article is distributed under the terms of the Creative Commons Attribution License (CC-BY 4.0), which permits any use, distribution and reproduction in any medium, provided the original author(s) and source are credited.

\section{References}

[1] J.M. Maldacena, Eternal black holes in Anti-de Sitter, JHEP 04 (2003) 021 [hep-th/0106112] [INSPIRE].

[2] M. Van Raamsdonk, Building up spacetime with quantum entanglement, Gen. Rel. Grav. 42 (2010) 2323 [Int. J. Mod. Phys. D 19 (2010) 2429] [arXiv:1005.3035] [INSPIRE].

[3] M. Van Raamsdonk, A patchwork description of dual spacetimes in AdS/CFT, Class. Quant. Grav. 28 (2011) 065002 [INSPIRE].

[4] J. Maldacena and L. Susskind, Cool horizons for entangled black holes, Fortsch. Phys. 61 (2013) 781 [arXiv:1306.0533] [InSPIRE].

[5] B. Swingle, Entanglement renormalization and holography, Phys. Rev. D 86 (2012) 065007 [arXiv: 0905.1317] [INSPIRE].

[6] G. Evenbly and G. Vidal, Tensor network states and geometry, J. Stat. Phys. 145 (2011) 891 [arXiv:1106.1082].

[7] B. Swingle, Constructing holographic spacetimes using entanglement renormalization, arXiv:1209.3304 [INSPIRE].

[8] X.-L. Qi, Exact holographic mapping and emergent space-time geometry, arXiv:1309.6282 [INSPIRE].

[9] J.I. Latorre and G. Sierra, Holographic codes, arXiv: 1502.06618 [INSPIRE].

[10] F. Pastawski, B. Yoshida, D. Harlow and J. Preskill, Holographic quantum error-correcting codes: Toy models for the bulk/boundary correspondence, JHEP 06 (2015) 149 [arXiv: 1503.06237] [INSPIRE].

[11] T. Hartman and J. Maldacena, Time evolution of entanglement entropy from black hole interiors, JHEP 05 (2013) 014 [arXiv:1303.1080] [INSPIRE].

[12] L. Susskind, Computational complexity and black hole horizons, arXiv:1403.5695 [arXiv: 1403.5695] [INSPIRE].

[13] D. Stanford and L. Susskind, Complexity and shock wave geometries, Phys. Rev. D 90 (2014) 126007 [arXiv:1406.2678] [INSPIRE].

[14] L. Susskind and Y. Zhao, Switchbacks and the bridge to nowhere, arXiv:1408.2823 [INSPIRE].

[15] L. Susskind, Entanglement is not enough, arXiv:1411.0690 [INSPIRE].

[16] L. Susskind, The typical-state paradox: diagnosing horizons with complexity, arXiv: 1507.02287 [INSPIRE].

[17] M. Miyaji, T. Numasawa, N. Shiba, T. Takayanagi and K. Watanabe, Gravity dual of quantum information metric, arXiv:1507.07555 [INSPIRE].

[18] A.R. Brown, D.A. Roberts, L. Susskind, B. Swingle and Y. Zhao, Complexity equals action, arXiv: 1509.07876 [INSPIRE]. 
[19] D. Marolf and J. Polchinski, Gauge/gravity duality and the black hole interior, Phys. Rev. Lett. 111 (2013) 171301 [arXiv:1307.4706] [INSPIRE].

[20] V. Balasubramanian, M. Berkooz, S.F. Ross and J. Simon, Black holes, entanglement and random matrices, Class. Quant. Grav. 31 (2014) 185009 [arXiv:1404.6198] [INSPIRE].

[21] J.L.F. Barbon and E. Rabinovici, Geometry And Quantum Noise, Fortsch. Phys. 62 (2014) 626 [arXiv:1404.7085] [INSPIRE].

[22] M. Alishahiha, Holographic complexity, arXiv:1509.06614 [INSPIRE].

[23] R. Emparan, AdS/CFT duals of topological black holes and the entropy of zero energy states, JHEP 06 (1999) 036 [hep-th/9906040] [INSPIRE].

[24] J.P.S. Lemos, Cylindrical black hole in general relativity, Phys. Lett. B 353 (1995) 46 [gr-qc/9404041] [INSPIRE].

[25] J.P.S. Lemos, Two-dimensional black holes and planar general relativity, Class. Quant. Grav. 12 (1995) 1081 [gr-qc/9407024] [INSPIRE].

[26] S. Aminneborg, I. Bengtsson, S. Holst and P. Peldan, Making Anti-de Sitter black holes, Class. Quant. Grav. 13 (1996) 2707 [gr-qc/9604005] [InSPIRE].

[27] R.B. Mann, Pair production of topological Anti-de Sitter black holes, Class. Quant. Grav. 14 (1997) L109 [gr-qc/9607071] [INSPIRE].

[28] R.-G. Cai and Y.-Z. Zhang, Black plane solutions in four-dimensional space-times, Phys. Rev. D 54 (1996) 4891 [gr-qc/9609065] [InSPIRE].

[29] D.R. Brill, J. Louko and P. Peldan, Thermodynamics of $(3+1)$-dimensional black holes with toroidal or higher genus horizons, Phys. Rev. D 56 (1997) 3600 [gr-qc/9705012] [InSPIRE].

[30] L. Vanzo, Black holes with unusual topology, Phys. Rev. D 56 (1997) 6475 [gr-qc/9705004] [INSPIRE].

[31] D. Birmingham, Topological black holes in Anti-de Sitter space, Class. Quant. Grav. 16 (1999) 1197 [hep-th/9808032] [INSPIRE].

[32] D. Birmingham and M. Rinaldi, Brane world in a topological black hole bulk, Mod. Phys. Lett. A 16 (2001) 1887 [hep-th/0106237] [INSPIRE].

[33] D. Birmingham and S. Mokhtari, Stability of topological black holes, Phys. Rev. D 76 (2007) 124039 [arXiv:0709.2388] [InSPIRE].

[34] D. Marolf, M. Rangamani and M. Van Raamsdonk, Holographic models of de Sitter QFTs, Class. Quant. Grav. 28 (2011) 105015 [arXiv: 1007.3996] [INSPIRE].

[35] S. Ryu and T. Takayanagi, Holographic derivation of entanglement entropy from AdS/CFT, Phys. Rev. Lett. 96 (2006) 181602 [hep-th/0603001] [INSPIRE].

[36] S. Ryu and T. Takayanagi, Aspects of holographic entanglement entropy, JHEP 08 (2006) 045 [hep-th/0605073] [INSPIRE].

[37] S.A. Hartnoll, Lectures on holographic methods for condensed matter physics, Class. Quant. Grav. 26 (2009) 224002 [arXiv:0903.3246] [INSPIRE].

[38] J.L.F. Barbon and C.A. Fuertes, A note on the extensivity of the holographic entanglement entropy, JHEP 05 (2008) 053 [arXiv:0801.2153] [INSPIRE].

[39] J.L.F. Barbón and J. Martínez-Magán, Spontaneous fragmentation of topological black holes, JHEP 08 (2010) 031 [arXiv: 1005.4439] [INSPIRE]. 
[40] G. Horowitz, A. Lawrence and E. Silverstein, Insightful D-branes, JHEP 07 (2009) 057 [arXiv:0904.3922] [INSPIRE].

[41] A. Bernamonti and B. Craps, D-brane potentials from multi-trace deformations in AdS/CFT, JHEP 08 (2009) 112 [arXiv:0907.0889] [INSPIRE].

[42] J.L.F. Barbon and E. Rabinovici, Holography of AdS vacuum bubbles, JHEP 04 (2010) 123 [arXiv: 1003.4966] [INSPIRE].

[43] D. Harlow, Metastability in Anti de Sitter space, arXiv:1003.5909 [INSPIRE].

[44] J.M. Maldacena, The large- $N$ limit of superconformal field theories and supergravity, Int. J. Theor. Phys. 38 (1999) 1113 [Adv. Theor. Math. Phys. 2 (1998) 231] [hep-th/9711200] [INSPIRE].

[45] S.S. Gubser, I.R. Klebanov and A.M. Polyakov, Gauge theory correlators from noncritical string theory, Phys. Lett. B 428 (1998) 105 [hep-th/9802109] [INSPIRE].

[46] E. Witten, Anti-de Sitter space and holography, Adv. Theor. Math. Phys. 2 (1998) 253 [hep-th/9802150] [INSPIRE]. 